\title{
1146+111B,C: A GIANT GRAVITATIONAL LENS?
}

\author{
E.L. Turner \\ Princeton University Observatory \\ Peyton Hall \\ Princeton, NJ 08544 \\ USA
}

\begin{abstract}
The history and current observational status of the giant gravitational lens candidate $1146+111 \mathrm{~B}, \mathrm{C}$ are reviewed. In the absence of any new positive evidence and in the presence of reported differences in the UV and IR spectra of B and C, the lens hypothesis is clearly much weaker than previously. Nevertheless, given the substantial similarity of the spectra over a broad range of wavelengths, discrepancies among various spectroscopic observations of $\mathrm{C}$, and the possibility of spectral variability plus large differential time delays, the data do not yet support any definite conclusion as to the nature of $1146+111 \mathrm{~B}, \mathrm{C}$.
\end{abstract}

\section{BACKGROUND AND INTRODUCTION}

Considerable attention has been attracted recently by $1146+111 \mathrm{~B}, \mathrm{C}$, a gravitational lens candidate with an unexpected and unprecedentedly large image separation of 157". If this system is actually a gravitational lens, it has important implications for large scale structure and dark mass in the Universe.

The two quasars in question were originally identified by $C$. Hazard on prism plates among a "concentration" of quasars at a variety of redshifts in the $1146+111$ field. This "concentration" was discussed by Hazard and his collaborators H. Arp and D. Morton in a pair of papers $(1979,1980)$. While noting the similarity of the redshift and spectra of B and C, Arp and Hazard (1980) dismissed a gravitational lens explanation on the grounds of an implausibly large redshift.

Several years later, Paczynski (1986) again called attention to the B,C pair as a possible example of a lensing effect by a hypothetical cosmic string.

In March 1986 we obtained very high signal-to-noise, moderate resolution spectra of $B$ and $C$ in the visual and red; we also obtained deep CCD images of the field. Examination of these data convinced us that they strongly supported the lensing hypothesis and that it should be taken more seriously; thus, we again called attention to the system in the literature (Turner et al. 1986). The primary evidence which 
influenced us was the very striking similarity of our spectra of the two objects. This similarity included the indistinguishable redshifts (based on the Mg II line), the nearly identical profiles and widths of the Mg II line, the similar strengths of the Fe II multiplet blends producing various bumps and wiggles in the "continuum", and the absence of [0 II] emission in both objects. Although our data did show statistically significant (but quite small) differences between the two objects, these could be accounted for in various ways (e.g., differential time delays plus variability) and seemed much less impressive than the remarkable similarities. It was our opinion that such a degree of similarity would be quite rare in quasars paired at random, even discounting the redshift agreement which could be explained by physical association. Certainly, no other quasar in our library of spectra generated in other programs has line shapes, widths, and relative strengths which would agree with those of $1146+111 \mathrm{~B}, \mathrm{C}$ nearly as well as they agree with one another. A secondary bit of evidence supporting the lens hypothesis for $1146+111 \mathrm{~B}, \mathrm{C}$ which influenced us was their association in the sky with several other quasars at various redshifts; this could have been understood rather naturally as the result of lens magnification of all background objects in the field.

\section{CURRENT OBSERVATIONAL SITUATION}

Since the appearance of our spectra of $1146+111 \mathrm{~B}, \mathrm{C}$ in the 1iterature, there have been several attempts to test the lens hypothesis by extending the spectroscopic comparison into the UV and IR and by searches for perturbations of the microwave background. Some of these have been published, some are in preprint form, some have been communicated privately, some we have carried out ourselves, and some may be as yet unknown to me. None of these new observations offer strong confirmation of the lens hypothesis (as they might have), and several seem to suggest that separate, physically associated objects are more likely. On balance, the case for a giant gravitational lens in $1146+111 \mathrm{~B}, \mathrm{C}$ is much weaker than when only our original spectra were available; nevertheless, in my opinion, the question is still an open one. All of the observational results currently known to me are briefly reviewed below:

Two efforts have been made to detect microwave background distortions associated with hypothetical lensing objects in $1146+111$, one by Stark et al. (1986) and the other by Lawrence et al. (1986). The former shows no fluctuations larger than about $1 \mathrm{mK}$ in a strip of sky running through the field while the latter reports no deviations larger than a few tenths of a $\mathrm{mK}$ at three selected positions in the field. Both results suggest that there is less hot gas between $B$ and $C$ than would normally have been expected for a massive galaxy cluster and limit the transverse velocity of any cosmic string passing between $B$ and $C$ to less than a few tenths of $C$.

Deep VLA maps of the field show no sources to limits of somewhat less than $0.1 \mathrm{mJy}$. This is not surprising given the fact that the quasars in the field were optically selected and the field of view of 
the VLA. Of course, if one or both of $B$ and $C$ had been a radio source, this would have been quite an important constraint.

Optical and near optical spectra have proliferated. I am aware of Arp and Hazard's (1980) published spectra (low resolution and $S / N$ ), our published KPNO spectra, Shaver and Cristiani's (1986) published IR spectra, spectra obtained by Huchra (1986) on 4 separate occasions at the MMT which extend we11 into the UV in three cases, spectra extending from the near UV out to 10,000§ obtained by Neugebauer and Soifer (1986) at the Hale 5-meter, and independent 5-meter IR spectra obtained with the 4-Shooter spectrograph by Gunn (1986). The interpretation of these spectra taken as a whole is far more uncertain and confusing than when they are considered individually. The following general remarks may be made: 1) Some pairs of spectra show a striking similarity between $B$ and C (e.g., our published spectra). 2) Some pairs show significant differences, particularly in Balmer line emission (Shaver and Cristiani) and C III] strength (Huchra's spectra). 3) Spectra obtained of $1146+111 \mathrm{~B}$ at different epochs and by different groups appear fairly consistent. 4) There are discrepancies between spectra of $1146+111 \mathrm{C}$ obtained at different epochs and by different groups. This last point deserves some elaboration. The two sets of Palomar spectra which extend into the IR both show Balmer emission in $\mathrm{C}$ as well as $\mathrm{B}$, thus contradicting the published IR spectra; on the other hand, they do not show Balmer lines of identical strength or width in B and C. Huchra's spectra obtained at different epochs appear inconsistent as to the strength and width of the C III] line. Huchra's spectrum shows a substantially broader $\mathrm{Mg}$ II line in $\mathrm{C}$ than either the published KPNO spectrum or the Neugebauer and Soifer spectrum (which agrees very well with the KPNO spectrum). The relative strength of Fe II blends in some of Huchra's spectra of $\mathrm{C}$ appear much stronger than in other spectra both by Huchra and others. Letme reiterate that these discrepancies apply to various observations of the same lines in the same object!

\section{DISCUSSION}

Although many have reached relatively definite conclusions (of various signs) as to whether or not $1146+111 \mathrm{~B}, \mathrm{C}$ is a gravitational lens or a pair of physically associated objects, I do not believe that any certain conclusion can be supported by the available data. Clearly the negative evidence of reported spectroscopic differences and, perhaps equally seriously, the absence of new positive evidence makes the case much weaker than when only the KPNO spectra were under discussion. Nevertheless, the lens hypothesis is no more logically excluded by the present data than the physically distinct objects hypothesis was by the earlier data. Indeed, given the physical possibilities of neighboring twin quasars on the one hand and variability plus long differential time delays on the other, no definitive test is possible solely based on spectroscopic similarities or differences. Given the (so far unexplained) difficulty in obtaining consistent spectra of $\mathrm{C}$, cautious interpretation seems even more necessary.

The case of $1146+111$ raises a number of important questions 
whether or not it is eventually shown to be due to a gravitational lens: If it is a lens, one may ask

1) What is the lensing object, or at least, how may its properties be constrained?

2) Are there other examples of such giant lens systems, and if so, how many and how may they be located?

If it is not a lens, one needs to ask

1) What is the explanation of the very great similarity of the spectra near Mg II: chance or somehow the result of physical association?

2) If these are not lensed, are there other objects among the "known" gravitational lenses (most of which have less similar spectra) which are also physical pairs?

3) What would constitute a more reliable test than spectral similarity?

4) Quantitatively, how much variation is there in the spectra of quasars of similar luminosity and redshift?

J.E. Gunn, J.P. Huchra, C.R. Lawrence, G. Neugebauer, D.P. Schneider, T. Soifer, and A. Stark have all generously communicated results in advance of publication. Those listed above and numerous other colleagues including particularly B.F. Burke, J.R. Gott, J.N. Hewitt, J.P. Ostriker, B. Paczynski, M. Schmidt, and A. Tyson have also provided helpful comments and insights concerning 1146+111. This work was supported in part by NSF grant AST84-20352.

\section{References}

Arp, H., and Hazard, C. 1980, Ap. J., 2440, 726.

Gunn, J.E. 1986, private communication:--

Hazard, C., Arp, H., and Morton, D.C. 1979, Nature, 282, 271.

Huchra, J.P. 1986, private communication.

Lawrence, C.R., Readhead, A.C.S., Moffet, A.T., and Birkinshaw, M. 1986, A.J., in press.

Neugebauer, G., and Soifer, T. 1986, private communication.

Paczynski, B. 1986, Nature, 31 $\underline{\underline{1}}$, 567.

Shaver, P.A., and Cristiani, S. 1986, Nature, 321, 585.

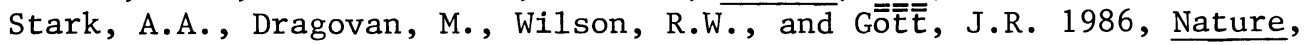
in press.

Turner, E.L., Schneider, D.P., Burke, B.F., Hewitt, J.N., Langston, G. I., Gunn, J.E., Lawrence, C.R., and Schmidt, M. 1986, Nature, 321 142 .

\section{DISCUSSION}

ARP: This pair is a member of a group of quasars which is very similar to other tight groups of quasars at about $z \simeq 1$ mean redshift. It is characteristic of these groups (see Arp, Liege Symposium 1983) that they have pairs which are very similar in redshift. But that similarity is generally only within 0.1 to 0.2 in redshift. This is direct evidence that quasars exist in physically similar pairs and that gravitational lenses are not necessarily demonstrated by quasars of similar spectra. 
TURNER: I agree that many lines of evidence, not just spectroscopy, must be mustered to "demonstrate" lensing in a particular case. I would be surprised if lensing played any role in general in the associations you mention.

WAMPLER: I want to disagree with the concept that these two spectra of $1146+111$ are "remarkably similar". Quasars, like people, are similar to each other and some are very similar. Much more detail is needed before it can be claimed that a "fingerprint" has been found. In this case the structure near MgII $\lambda 2800$ is due to the multiplet structure of FeII, and not to similar intensities of independent emission lines.

TURNER: I certainly agree that much of the structure near MgII is due to FeII and that there are many fewer free physical parameters

describing the gas than independent points in our spectra. Nevertheless, I do find the agreement "remarkable". I would include the similar Mg II to Fe II relative intensities, the nearly identical profile shapes in $\mathrm{Mg}$ II, the identical (within the measurement errors) Mg II line widths, and the absence of other lines, notably [0II] in both objects. We have examined a sample of $\sim 20$ quasars with $z \simeq 1$ and found no pair which match so precisely.

BURKE: This is a comment that in part is a summary of the positive aspects of the gravitational lens situation, which has some elements of confusion at present. The gravitational lens phenomenon is important because we know that reliable examples exist; lensing may provide the most reliable tracer for dark matter on a large scale, their frequency of occurrence sets hard lower limits on $\Omega$, as J. Hewitt has shown, and the time delay gives information on $\delta \rho / \rho$, as Alcock and Anderson have discussed, if there is a good value for $\mathrm{H}_{0}$. Therefore, the criteria for identification should be clear and stringent. These should be, in my opinion, an appropriate subset (preferably seven) of the following: (1) Repetition of flux variations with a well-measured time delay. This is sufficient proof, but has not yet been reliably achieved for any lensed quasars. (2) Similar spectra are needed, of course. If there are differences in the spectra, there must be a good explanation $(0957+561$ is a good example of differences being convincingly explained). (3) Similar milliarcsecond structure (from VLBI at present, from optical interferometry at some future time). Again differences must be explained. Relative parity is a powerful proof (the VLBI work of Gorenstein et al. on $0957+561$ is a good example). (4) Large-scale structure gives much tighter requirements on the lens. Examples of the use of this criterion is given by the radio jet structure of 0957+561, the third image of 2016+112, and the splitting of $A$ into $A_{1}$ and $A_{2}$ in $1115+080$ (which turned the 
triple quasar into a quadruple quasar, which actually should be a quintuple quasar). Note that the radio selection method is a neutral one, having the advantage of allowing all four tests. I suggest that we have 2 classes of lens candidates: Class A, which are wellestablished and for which the burden of proof is on the challenger $(0957+561,1115+080,2016+112$, and $2237+035$ are the four present examples of Class A lenses); and Class B lenses, which meet the criteria to some degree, but which still lay the burden of proof upon the observers, with further evidence needed ( $1146+111$ is certainly in this category). Some have suggested that one should see visible evidence of the lens itself, but in our current state of knowledge (or lack of knowledge) of dark matter, I suggest that this is not a necessary requirement, or at least not yet. 